\title{
High-temperature hybrid phthalonitrile/amino-MMT nanocomposites: Synthesis, structure, properties
}

\author{
V. A. Bershtein ${ }^{1}$, A. M. Fainleib ${ }^{2 *}$, P. N. Yakushev ${ }^{1}$, D. A. Kirilenko ${ }^{1}$, K. G. Gusakova ${ }^{2}$, \\ D. A. Markina ${ }^{1}$, O. G. Melnychuk ${ }^{2,3}$, V. A. Ryzhov ${ }^{1}$ \\ ${ }^{1}$ Ioffe Institute, 194021 St.-Petersburg, Russia \\ ${ }^{2}$ Institute of Macromolecular Chemistry, 02160 Kyiv, Ukraine \\ ${ }^{3}$ National University of 'Kyiv-Mohyla Academy', 04655 Kyiv, Ukraine
}

Received 21 December 2018; accepted in revised form 25 February 2019

\begin{abstract}
Hybrid nanocomposites based on heterocyclic network, obtained from bisphenol A based phthalonitrile (BAPhN) with different (0.03-5.0 wt \%) contents of reactive amino-montmorillonite (MMT) nanolayers, were synthesized and studied for the first time. Their structure, dynamics, thermal, relaxation and elastic properties were characterized using transmission electron microscopy (TEM), mid-infrared (mid-IR), far-infrared (far-IR) and energy dispersive X-ray (EDX) spectroscopies, differential scanning calorimetry (DSC), and by dynamic mechanical analysis (DMA) and thermogravimetry (TGA) measurements performed in both air and nitrogen mediums at temperatures from 20 to $600-900^{\circ} \mathrm{C}$. Depending on nanofiller content, different extents of MMT stacks exfoliation, from single nanolayers to MMT stacks with tens nanolayers-thickness, are observed in the nanocomposites. The pronounced dynamic heterogeneity in the glass transition and the 'constrained dynamics' effects are shown. For the pristine matrix, $T_{\mathrm{g}}$ (DMA) $=446^{\circ} \mathrm{C}$ varying from 460 to $570{ }^{\circ} \mathrm{C}$ for the nanocomposites. After high-temperature treatment in $\mathrm{N}_{2}$ medium, the relaxation spectrum and glass transition disappear, and constant dynamic modulus $E^{\prime} \approx 3 \mathrm{GPa}$ at $20-600^{\circ} \mathrm{C}$ is registered. A satisfactory thermal stability of the nanocomposites, with retaining the sample integrity is observed at temperatures up to $\sim 500^{\circ} \mathrm{C}$ in air and up to $900{ }^{\circ} \mathrm{C}$ in $\mathrm{N}_{2}$ medium.
\end{abstract}

Keywords: nanocomposites, phthalonitrile/amino-MMT, synthesis, structure, properties

\section{Introduction}

Polymer nanocomposites have attracted enormous attention in both academia and industry over the past decades. Their excellent properties are attributed to the large surface/volume ratio of the nanofillers. Various types of 3-D, 2-D or 1-D nanofillers with various sizes and shapes (particles, platelets, wires, fibers, rods, etc.) have been used to prepare polymer nanocomposites [1-4]. However, the exfoliated layered silicates (clays) have been used especially widely as nanofillers in polymer nanocomposites. Easy accessibility in nature, low cost, environmentally friendly properties and high aspect ratio of silicate 2-D nanolayers are amongst the greatest advantages of clay particles over the other nanofillers to promote their use in nanocomposites $[4,5]$.

Demand for polymeric materials with high thermal stability continues to be increasingly important in some fields, for instance, in aircraft, space and marine structures, in 'hot zones' of different constructions and in microelectronics. The combination of nanoparticles and a high-temperature resistant polymer can contribute to the advanced composites by further improving their performance. With the advancement of technologies and to broaden the applications of composites in aircraft and other structures, the composite systems which can be used in the temperature range of $300-400^{\circ} \mathrm{C}$ and higher are needed. 
In order to meet these demands, several classes of high performance thermosetting matrices have been developed in the last decades. Such matrices possess usually excellent thermal and thermo-oxidative resistance, high dimensional stability at elevated temperatures, low water absorption, good chemical resistance and high mechanical properties. Cyanate ester resins (CER), bismaleimides, benzoxazines and bisphthalonitrile (BPhN)-based heterocyclic networks are best known amongst the widely used high temperature-resistant thermosets.

To our knowledge, the first attempts on synthesis and investigation of CER-based nanocomposites with both virgin and organically modified montmorillonite (MMT) clay have been described in [6-9]. Introduction of 10-20 wt $\%$ of modified clay dramatically affected the flammability properties. Ganguli and coworkers $[10,11]$ found that $2.5-5 \mathrm{wt} \%$ of organically-modified MMT improved thermal properties (coefficient of thermal expansion, $T_{\mathrm{g}}$ and thermal stability) as well as increased elastic modulus and mechanical strength by $\sim 30 \%$. The curing behavior and rheological characteristics of CER/organoclay systems were studied by Ganguli et al. [11], Wooster et al. [12] and Kim et al. [13]. Catalytic effect of different organo-modified clays on CER polycyclotrimerization and improvement of strength, cracking resistance and thermal characteristics of the final nanocomposites have also been described [12-21]. In previous works $[22,23]$, we reported on the synthesis and investigation of polymerization kinetics, morphology, molecular mobility, thermal and mechanical properties of CER-based nanocomposites containing partially exfoliated organo-MMT with $\mathrm{OH}$ groups on the surface. Recently [24] nanocomposites were synthesized and studied which were based on densely cross-linked CER doped by aminoMMT nanolayers. Ultralow MMT additives, covalently embedded into the CER network, exerted an anomalously large impact on its properties, in particular, increasing the onset of glass transition temperature $T_{\mathrm{g}}$ by $40^{\circ} \mathrm{C}$ and providing two-fold rise of modulus at $20-200^{\circ} \mathrm{C}$. The idea about the extraordinarily enhanced long-range action of the constrained dynamics effect in the case of densely crosslinked polymer networks was suggested [24].

Bisphthalonitrile $(\mathrm{BPhN})$ polymer networks and composites based thereon are of special interest as the new generation of high-temperature materials for the applications under extreme conditions [25, 26]. Last years, they gained the leading place amongst the high-performance polymers thanks to their unique thermal performance and superior flame resistance, low water uptake, advanced UV shielding, excellent chemical resistance, etc. Keller and coworkers patented [27] bisphthalonitrile resins, cross-linked polymers and composites thereof and published the first papers $[28,29]$ on the synthesis and characterization of $\mathrm{BPhN}$-based thermosetting polymers.

The most effective method of further enhancing properties of $\mathrm{BPhN}$ polymers is synthesis of nanocomposites. Only recently, the first publications on phthalonitrile nanocomposites appeared in literature [30-36]. Lei and coworkers [30, 31] synthesized the nanocomposites with 5-10 wt \% exfoliated graphite nanoplatelets and improved flexural strength, modulus and thermal stability. Derradji and coworkers [32-35] synthesized the phthalonitrile nanocomposites with silicon nitride [32], ceramics [33] and surface-modified $\mathrm{ZnO}$ [34] or titania [35] nanoparticles. The substantial effects of introducing nanoparticles for enchancing thermal and mechanical properties of $\mathrm{BPhN}$-based matrix have been shown. For example, dynamic modulus $E^{\prime}$ and $T_{\mathrm{g}}$ increased up to $4 \mathrm{GPa}$ and $360^{\circ} \mathrm{C}$, respectively [32]. Li et al. [36] synthesized and studied phthalonitrile-polyhedral oligomeric silsesquioxane (POSS) nanocomposites.

Although the first publications on phthalonitrile nanocomposites indicated their advantages regarding the other thermostable materials, the fundamental studies in this field are almost absent up to now. Recently [37], we have performed the combined research of a series of nanocomposites based on bisphenol A bisphthalonitrile (BAPhN) network containing $0.5 \mathrm{wt} \%$ of different reactive, amino- or epoxy-functionalized POSS nanoparticles and MMT nanolayers. Their structure, dynamics, thermal, relaxation and elastic properties over the broad temperature range were characterized using TEM, mid-IR, far-IR and EDX spectroscopies, DSC, DMA and TGA techniques. The results obtained showed, in particular, the predominance of phthalocyanine macrocycles in the matrix network; the quasi-regular distribution of POSS nanoparticles in the amorphous matrix, and the satisfactory exfoliation of MMT stacks. $T_{\mathrm{g}}$ DMA) equaled to $380-390^{\circ} \mathrm{C}$ but increased up to 460 $560^{\circ} \mathrm{C}$ after post-curing by heating up to $430^{\circ} \mathrm{C}$.

In the present work, we have performed, for the first time, the combined research of a series of BAPhNbased nanocomposites with amino-MMT nanolayers 
at the wide variation of their content, from 0.03 to $5 \mathrm{wt} \%$.

\section{Experimental}

\subsection{Initial materials and synthesis of BAPhN/MMT nanocomposites}

Monomer, 2,2-bis[4-(3,4-dicyanophenoxy)phenyl] propane (bisphenol A phthalonitrile, BAPhN) $(>95 \%)$, was purchased from Shostka Chemical Reagents Plant, Ukraine. Amino-modified montmorillonite (amino-MMT, with 70-75\% MMT and 25-30\% Octadecylamine) under the trade names Nanomer ${ }^{\mathbb{B}}$ I.30E was supplied by Nanocor Inc., USA. N-Methyl2-pyrrolidone (NMP) was purchased from Merck, and methylethylketone (MEK) was supplied by $\mathrm{DutCH}_{2}$ (Netherlands). All the chemicals were used as received.

BAPhN was melted and heated at $T=200^{\circ} \mathrm{C}$ for 40 $50 \mathrm{~min}$. The BAPhN-based oligomer obtained and a mixture of organic solvents (NMP/MEK = $20 / 80 \mathrm{v} / \mathrm{v} \%$ ) were charged in the following ratio: $\mathrm{BAPhN} /(\mathrm{NMP} / \mathrm{MEK}=20 / 80 \mathrm{v} / \mathrm{v} \%)=60 / 40 \mathrm{wt} \%$ to a $50 \mathrm{ml}$ flask equipped with a mechanical stirrer and refluxing condenser, followed typically by heating for $40-45 \mathrm{~min}$ at $70^{\circ} \mathrm{C}$. Then, a calculated amount of the modified MMT nanoparticles was added and stirred for $15 \mathrm{~min}(1300 \mathrm{rpm})$ at the same temperature until a homogeneous solution was obtained. Next, the $\mathrm{BAPhN} /$ nanoparticles solution was poured into the mold with surface covered by anti-adhesive PTFE film, and cured in a programmable oven by step by step heating for $8 \mathrm{~h}$ at $260^{\circ} \mathrm{C}, 8 \mathrm{~h}$ at $280^{\circ} \mathrm{C}$, and $10 \mathrm{~h}$ at $300^{\circ} \mathrm{C}$. Synthesis of neat cured BAPhN matrix was carried out in the same way. The films of about $0.5 \mathrm{~mm}$ thickness were obtained.

However, as shown by IR spectroscopic analysis [37], the curing regime used during the synthesis resulted in only a partial polymerization of BAPhN, up to $83-89 \%$ for neat matrix and MMT-containing nanocomposites. Incomplete polymerization was conditioned by strongly suppressed mobility of densely cross-linked matrix network at $T \leq 300^{\circ} \mathrm{C}$ and high steric hindrances for completion of this reaction. Therefore, in [37] a series of post-curing procedures was performed at different temperatures from 340 to $530^{\circ} \mathrm{C}$ with estimating the attained polymerization degrees $(\mathrm{PD})$ of $-\mathrm{C} \equiv \mathrm{N}$ groups. It was shown that different post-curing regimes allowed increasing $\mathrm{PD}$ up to the limiting values of around $95 \%$. In this work, we prepared the post-cured samples by heating of synthesized samples up to $430^{\circ} \mathrm{C}$ with the rate of $3^{\circ} \mathrm{C} \cdot \mathrm{min}^{-1}$ (directly in the DMA setup, see below).

\subsection{Methods}

\subsubsection{Transmission electron microscopy combined with energy-dispersive X-ray spectroscopy}

The state and nanodistribution of MMT nanoparticles in the composites have been characterized by means of transmission electron microscopy (TEM) using Jeol JEM-2100F microscope (accelerating voltage $200 \mathrm{kV}$, conventional bright-field imaging mode, point-to-point resolution $0.19 \mathrm{~nm}$ ) equipped with Oxford Instruments INCA energy-dispersive X-ray spectrometer. The latter was used for the additional verification of observed structural features nature and controlling local elemental composition in the sample nanovolumes.

Because of brittleness of the materials under study, specimens for investigation by TEM were prepared by grinding the samples, using $30 \mu \mathrm{m}$ grit size silicon carbide sandpaper, to a powder; the latter was then dispersed in ethanol and poured on a conventional copper TEM grid covered with an ultrathin graphene oxide films [38]. The powder particles, thus obtained, have typically up to 5-10 $\mu \mathrm{m}$ size and are transparent for electrons only along their much thinner $(<100 \mathrm{~nm})$ edges. Measurements artefacts related to the use of the silicon carbide sandpaper are excluded because the silicon carbide debris are crystalline, possess a specific shape and provide a special diffraction contrast in TEM. This allowed distinguishing and excluding the silicon carbide particles from the analysis. It should be mentioned also that grinding did not lead to any exfoliation of MMT stacks inside the obtained microparticles of the nanocomposites; so, the morphology of the latter should remain rather intact if we consider those situated inside the matrix. It is noteworthy that the studied materials were rather stable under electron beam.

EDX spectra were obtained using electron beam focused to spots of ca. $2 \mathrm{~nm}$ in diameter. The application of EDX spectroscopy in addition to TEM has an advantage of higher spatial resolution of the technique down to a nanometer scale; that allowed, for example, direct verification of the presence of single MMT nanolayers ( $\mathrm{Si}, \mathrm{Al}$ and $\mathrm{Mg}$ elements) in the nanocomposites. 


\subsubsection{Mid- and far-infrared spectroscopy}

We used IR spectroscopy for getting the information on molecular structure of the nanocomposite matrix, polymerization control, and the changes in the matrix molecular dynamics caused by both post-curing and the incorporated MMT nanoparticles. Fourier transform infrared (FTIR) spectra were recorded using a Bruker model Tensor 37 spectrometer (Bruker AXS, Inc., Madison, WI, USA) between 4000 and $450 \mathrm{~cm}^{-1}$. For each measurement, optical-grade $\mathrm{KBr}$ pellets with the sufficient quantity of sample powders were prepared. Each spectrum comprising 32 consecutive scans with a resolution of $0.6 \mathrm{~cm}^{-1}$ was averaged. Polymerization (cross-linking) process was controlled by decreasing of the intensity for the absorption band of $-\mathrm{C} \equiv \mathrm{N}$ groups stretching vibrations with the maximum at $2226 \mathrm{~cm}^{-1}$.

Far-IR spectra were registered over the $20-300 \mathrm{~cm}^{-1}$ range using a FIS-21 Hitachi spectrometer (Hitachi Ltd., Japan) with a resolution of $1 \mathrm{~cm}^{-1}$. The film samples' thickness equaled about $100 \mu \mathrm{m}$. Three repeat measurements were performed in all cases, and it allowed registering the spectral maxima with the error of $\pm 0.5 \mathrm{~cm}^{-1}$.

\subsubsection{Differential scanning calorimetry, thermogravimetric analysis}

Differential scanning calorimetry (DSC) experiments were performed using a DSC 6300 Seiko Instruments apparatus (Seiko Instruments, Tokyo, Japan) for estimating glass transition temperature $T_{\mathrm{g}}$ at the heating rate of $20^{\circ} \mathrm{C} \cdot \mathrm{min}^{-1}$; the film sample mass was of about $30 \mathrm{mg}$.

Thermogravimetric analysis (TGA) and derivative thermogravimetry (DTG) were performed using a TGA Q50 TA Instruments under both air and inert (flowing nitrogen) atmospheres. Sample pellets with masses ranging from 10 to $20 \mathrm{mg}$ were heated from 20 to 700 or $900{ }^{\circ} \mathrm{C}$ with the heating rate of $20^{\circ} \mathrm{C} \cdot \mathrm{min}^{-1}$.

\subsubsection{Dynamic mechanical analysis}

Relaxation dynamics and elastic properties of the nanocomposites were estimated by dynamic mechanical analysis (DMA). The experiments were performed in the tensile mode using a DMS 6100 Seiko Instruments spectrometer (Seiko Instruments, Tokyo, Japan) at $1 \mathrm{~Hz}$ over the temperature range from 20 to $570{ }^{\circ} \mathrm{C}$, at the heating rate of $3{ }^{\circ} \mathrm{C} \cdot \mathrm{min}^{-1}$. The working part of test samples was of about
$20 \times 5 \times 0.5 \mathrm{~mm}^{3}$ size. The measurements were performed basically in air but also under $\mathrm{N}_{2}$ atmosphere. Two heating cycles were carried out typically for an each sample: the first cycle from 20 to $430^{\circ} \mathrm{C}$ characterized the properties of initially cured composites and performed, simultaneously, the role of post-curing procedure [37]. During the second heating cycle, from 20 to $570^{\circ} \mathrm{C}$, the characteristics of post-cured samples were estimated. This apparatus allowed carrying out the experiments up to about $570^{\circ} \mathrm{C}$ only. The third heating cycle was also performed in flowing nitrogen medium in the control experiments. Loss modulus $E^{\prime \prime}$, dynamic (storage) modulus $E^{\prime}$, and mechanical loss factor $\tan \delta=E^{\prime \prime} / E^{\prime}$ as the functions of temperature were measured. The glass transition temperatures, $T_{\mathrm{g}}$ 's, at $\tan \delta$ peaks maxima (or, sometimes, also at $E^{\prime \prime}$ peaks maxima) were determined.

\section{Results and discussion}

\subsection{Matrix molecular structure and dynamics as estimated by IR spectroscopy}

As it has been reported earlier [36, 39-45], the conversion of $\mathrm{BPhN}$ during polymerization might generate triazine, isoindoline, and phthalocyanine heterocycles in the network formed. However, there was no unambiguous description of the $\mathrm{BPhN}$ polymerization chemistry, obviously, because of some differences in polymer compositions, curing regimes, etc. Indeed, Li et al. [36] and Sheng et al. [39] indicated that the only polymerization product is polytriazine. Kumar et al. [40] found only polyisoindoline, and Zou et al. [41] found only phthalocyanine in the $\mathrm{BPhN}$ network formed. Wu et al. [42] described formation of triazine and phthalocyanine structures but did not reveal formation of isoindoline fragments. Ji et al. [43] assert formation of isoindoline and phthalocyanine structures without any traces of triazine cycles. Sheng et al. [44] reported that indoline and triazine structures were formed during $\mathrm{BPhN}$ polymerization. At last, according to [45], metal-free phthalocyanine fragments with the traces of triazine rings were formed. Anyway, it was natural to suppose, indeed, that the polymer networks produced from bisphthalonitrile monomer may exhibit complicated chemical structures containing heterocycles of different types in different ratios depending on different factors.

In our previous work [37], this problem was analyzed spectroscopically regarding the nanocomposites with 
the same matrix as herein and $0.5 \%$ POSS nanoparticles. It was shown, in particular, that the absorption band of reactive nitrile $-\mathrm{C} \equiv \mathrm{N}$ groups centered at around $2230 \mathrm{~cm}^{-1}$ sharply decreased in the course of polymerization but remained, to some extent, even after post-curing: the estimated limiting PD was around $95 \%$. Besides, mid-IR spectra showed the presence of phthalocyanine $\left(\sim 3400\right.$ and $\left.1010 \mathrm{~cm}^{-1}\right)$, triazine (1360 and $1520 \mathrm{~cm}^{-1}$ ) and, presumably only, isoindoline heterocycles $\left(1720 \mathrm{~cm}^{-1}\right)$ [37].

Figure 1 shows FTIR spectrum of the cured BAPhN/ MMT I.30E(5\%) nanocomposite in the mid-IR region. One can see, again, that the absorption band of nitrile groups, at $2226 \mathrm{~cm}^{-1}$, does not disappear totally. Thus, the most of $-\mathrm{C} \equiv \mathrm{N}$ groups only have participated in the polymerization reaction. This spectrum confirms the complex matrix structure and the opportunities for formation of different heterocycles in the structures of the cured matrix, namely, phthalocyanine (3420 and $\left.1012 \mathrm{~cm}^{-1}\right)$, triazine (1360 and $\left.1520 \mathrm{~cm}^{-1}\right)$, and, undoubtedly in this case, isoindoline heterocycles $\left(1730,1663\right.$, and $\left.1503 \mathrm{~cm}^{-1}\right)$.

Far-IR spectra of the BAPhN/POSS nanocomposite, presented in the previous work [37], showed that the

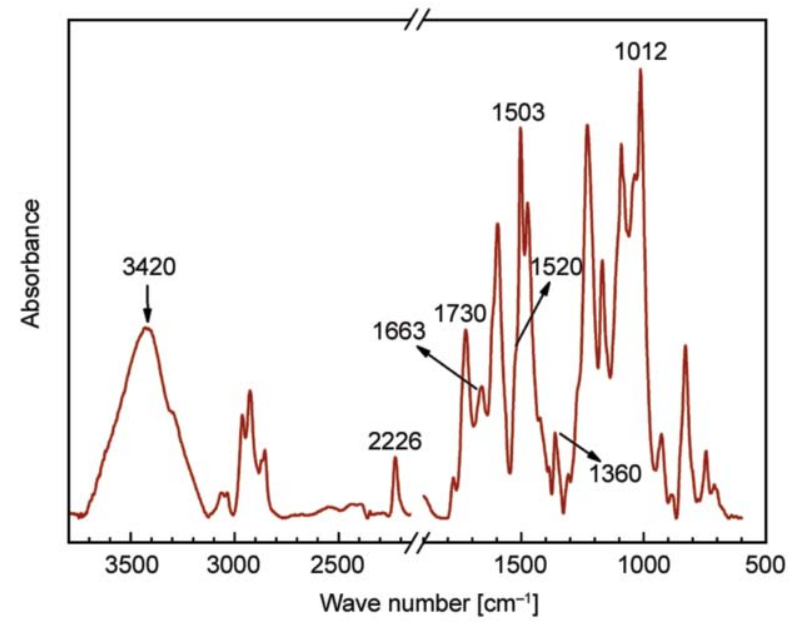

Figure 1. FTIR spectrum of the cured BAPhN/MMT I.30E (5\%) nanocomposite.

basic feature of its spectrum was the intense absorption band with the maximum at $155 \mathrm{~cm}^{-1}$ and some contributions of the bands with the maxima at 131, 182 and $224 \mathrm{~cm}^{-1}$. This allowed us to suppose the basic contribution of phthalocyanine macrocycles to the amorphous BAPh-based network. Indeed, according to Kobayashi [46], far-IR spectrum of crystalline phthalocyanine includes the absorption bands of deformation vibrations of these macrocycles with
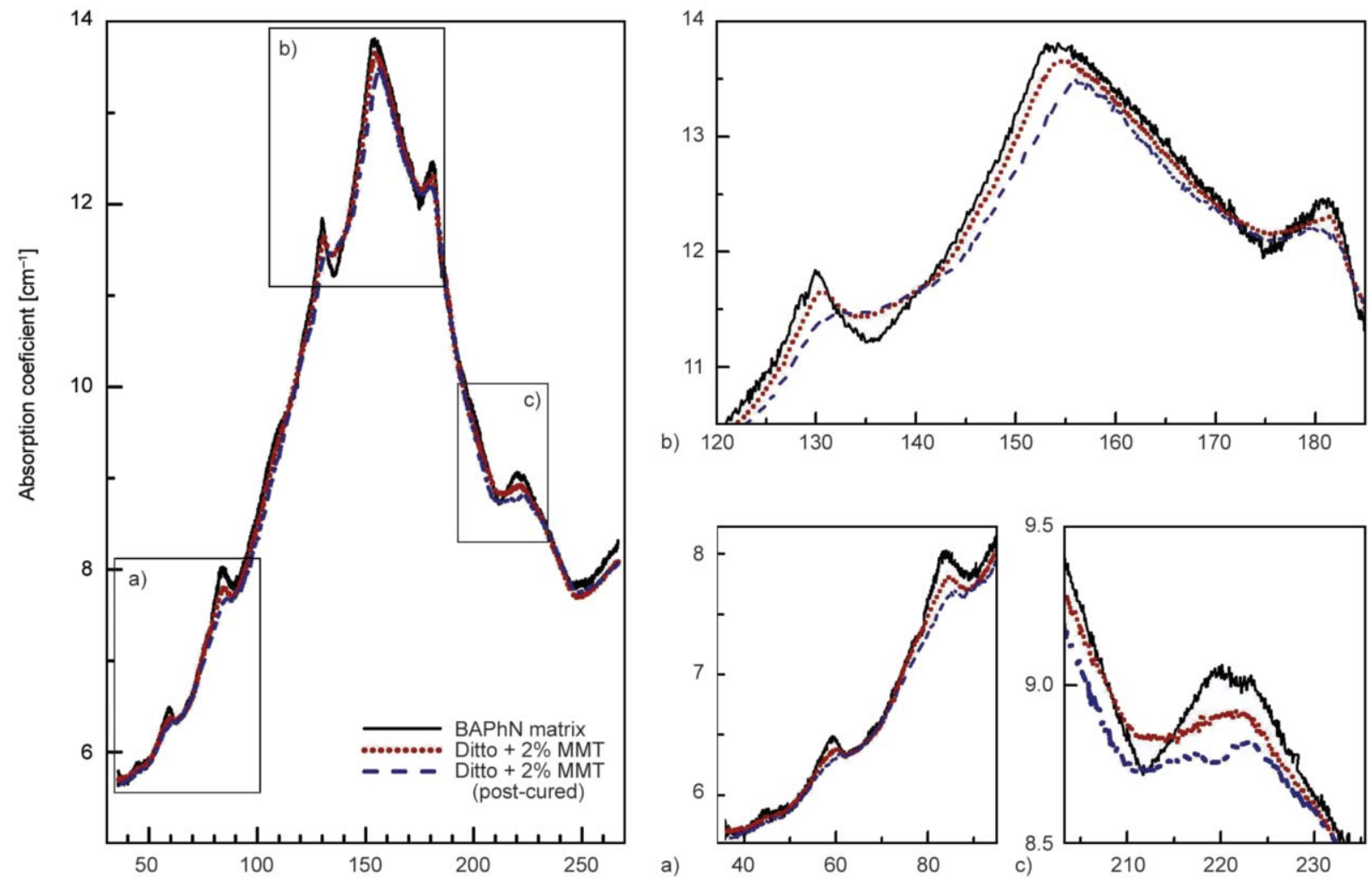

Wave number $\left[\mathrm{cm}^{-1}\right]$
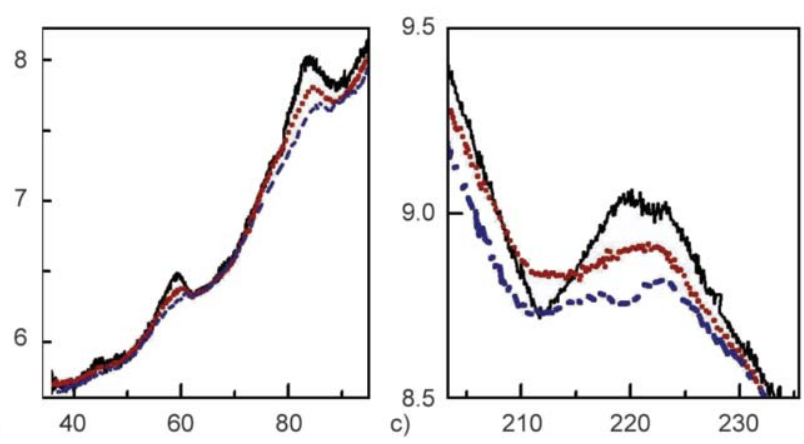

Figure 2. Far-IR spectra of the cured neat BAPhN matrix and BAPhN/MMT I.30E(2\%) nanocomposite in the cured and post-cured states (enlargements marked as a)-c)) 

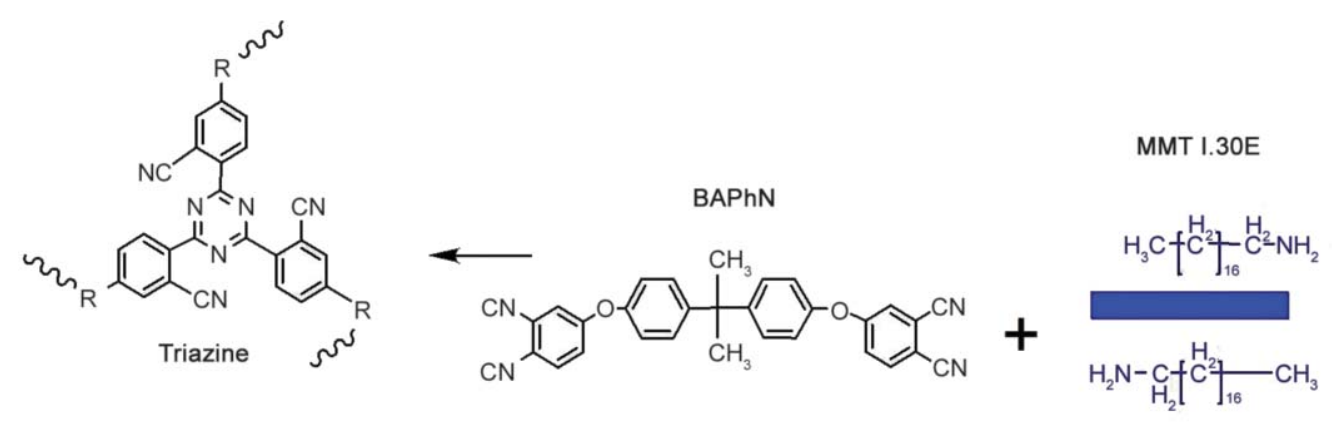

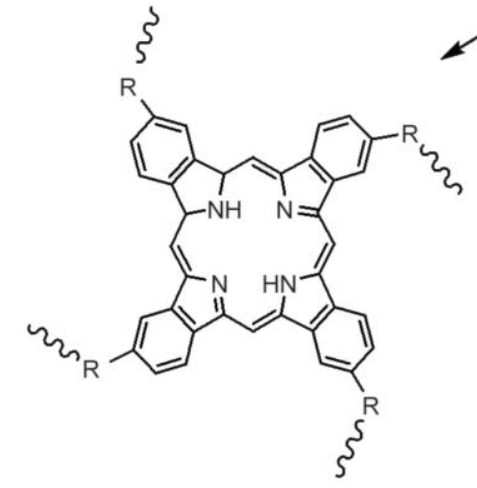

Phthalocyanine

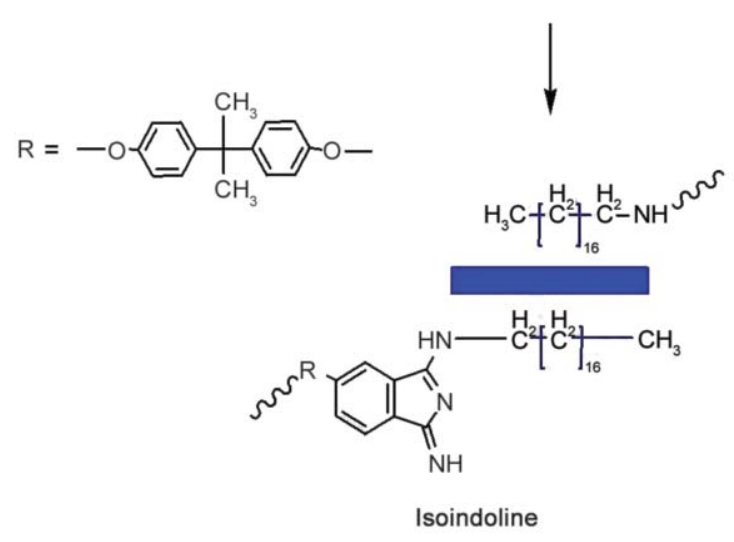

Figure 3. Schemes of polymerization reactions, formation of heterocycles in BAPhN network, and hybridization of the matrix with functionalized MMT I.30E nanoparticle.

the maxima at $155,127,140$ and $\sim 230 \mathrm{~cm}^{-1}$. Additionally, the spectrum of the cured BAPhN/POSS nanocomposite [37] included low intense absorption at $\sim 50$ to $120 \mathrm{~cm}^{-1}$ with the maximum at $84 \mathrm{~cm}^{-1}$ corresponding to small-angle vibrations ('librations') of triazine and benzene cycles [47-50]. Its low intensity might be originated from both the low content of triazine cycles and/or their strongly suppressed mobility in the BAPhN network.

Figure 2 shows the far-IR spectra of neat BAPhN matrix and the cured and post-cured samples of BAPhN-based nanocomposite with $2 \mathrm{wt} \%$ MMT. Again, the most intense absorption band at $155 \mathrm{~cm}^{-1}$ and some contributions of the bands with the maxima at $\sim 130,180$ and $220 \mathrm{~cm}^{-1}$ may be seen in the spectrum of the neat matrix because of the basic contribution of phthalocyanine macrocycles to the amorphous BAPhN network. Besides, this spectrum contains also low-intensity bands of triazine cycles vibrations at $\sim 54$ and $84 \mathrm{~cm}^{-1}$. Introducing of the nanofiller and post-curing procedure result in small but discernable changes in the spectrum: a decrease in absorption intensity, the displacement of the peak maxima by several $\mathrm{cm}^{-1}$ to higher wave numbers, and practically the disappearance of slight absorption peaks at frequencies below $100 \mathrm{~cm}^{-1}$. The latter may be originated from both the decreased content of triazine cycles and/or their strongly suppressed mobility in the BAPhN network. On the whole, these spectral changes correspond to constraining of network dynamics.

The chemical incorporation of the specifically functionalized nanoparticles into the matrix network took place, undoubtedly, because of high reactivity of $-\mathrm{C} \equiv \mathrm{N}$ groups regarding amine functional groups [28]. Figure 3 shows the schemes of polymerization reactions with formation of heterocycles in $\mathrm{BAPhN}$ network and the hybridization of the matrix with functionalized MMT I.30E nanoparticle.

\subsection{Nanostructure of nanocomposites}

Figure 4 shows the typical TEM images obtained for the nanocomposites with different MMT contents: $0.03,0.5,1$ and $5 \mathrm{wt} \%$. In each case a couple of images is given where the most typical nanostructure is shown on the left and more rarely registered nanostructure is shown on the right. One can see that these composites are characterized by very different levels of MMT dispersion (exfoliation) within the amorphous matrix depending on the MMT weight content. At $0.03 \%$ (and also at $0.1 \%$ ) MMT), the maximal degree of MMT exfoliation is attained when basically the single nanolayers or, rarely only, two-layer MMT formations are observed. The manifestation of 
a)
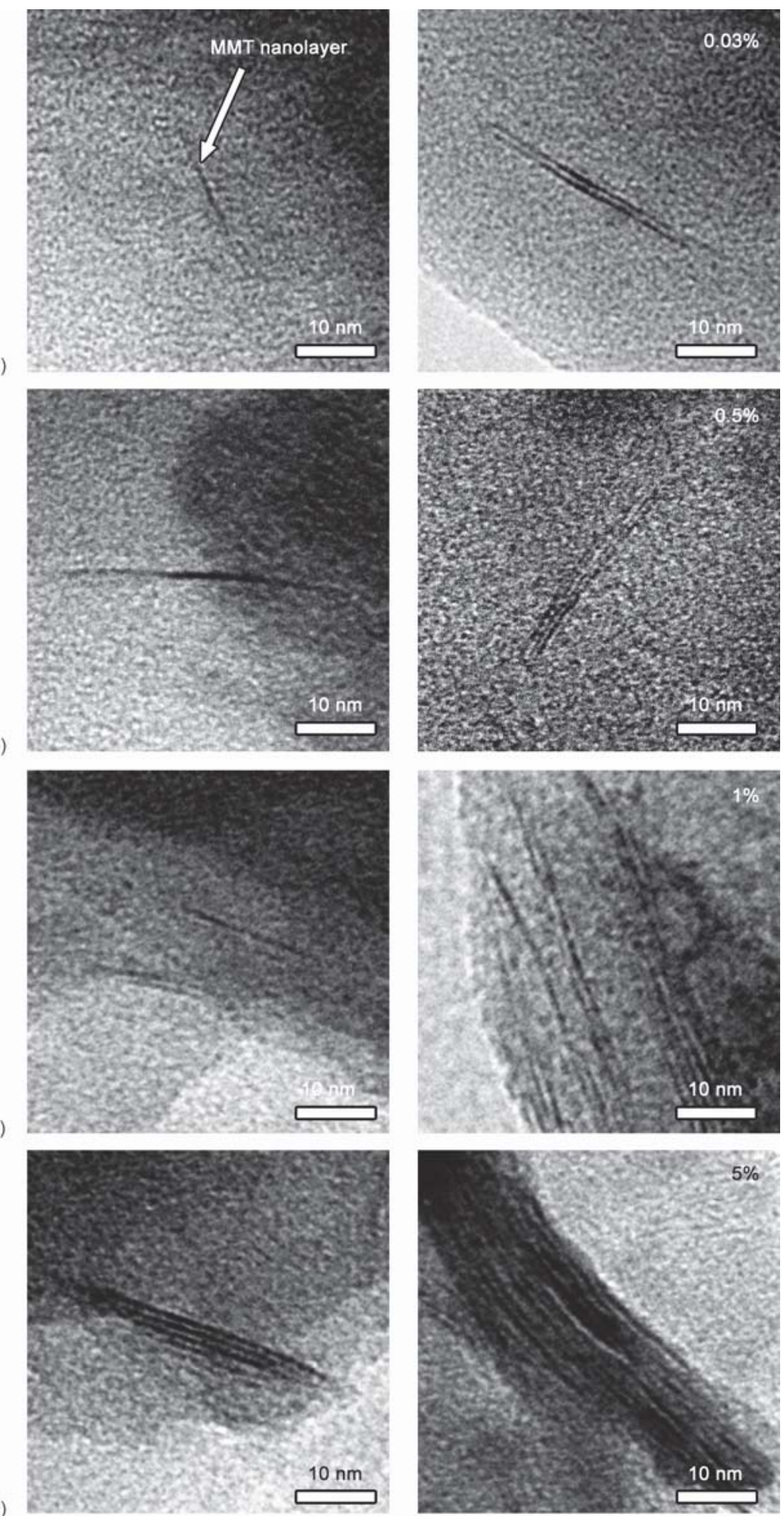

Figure 4. TEM micrographs obtained for the BAPhN/MMT 1.30E nanocomposites with different indicated contents of nanofiller. For an each composition typical images are shown on the left, and rarely registered ones are shown on the right, (a) $0,03 \%$, (b) $0,5 \%$, (c) $1 \%$, (d) $5 \%$

single nanolayers is also characteristic of the nanostructure for the composite with $0.5 \mathrm{wt} \% \mathrm{MMT}$ although sometimes the thin, three-four layer, MMT stacks may be seen as well. Very interesting TEM images were obtained for the nanocomposite with $1 \mathrm{wt} \%$ MMT. In this case, along with the most typical manifestation of one-two nanolayers formations in the matrix, one could observe also the more complex picture when a single MMT nanolayer, two-layers and five-ten layers MMT stacks can be seen simultaneously within $\sim 100 \mathrm{~nm}$ image (Figure 4 ). The exfoliation degree sharply decreased with increasing 
MMT content to $2 \mathrm{wt} \%$ and especially at $5 \mathrm{wt} \%$ MMT. In the former case, two-three layer MMT fragments prevailed in the nanostructure but the rather thick MMT stacks were also present. At last, in the nanocomposite with 5\% MMT relatively low level of MMT stacks exfoliation was observed, namely, basically the thin and rather thick MMT stacks only, with the thicknesses of ca. 5 and 20-30 nm, respectively, were formed (Figure 4).

Figure 5 shows, as an example, the typical EDX spectra, which were acquired, using electron beam focused to $2 \mathrm{~nm}$ spots, from the nanocomposite matrix, from a single MMT nanolayer, and from a MMT stack. These spectra allow tracking the basic features observed in the TEM image, e.g., to confirm that the seen dark lines correspond, indeed, to MMT nanolayer of about $1 \mathrm{~nm}$-thickness.

The spatial resolution of elemental composition estimation is determined with the diameter of the used electron beam. The indicated spot diameter exceeds the thickness of a MMT nanolayer, and therefore its spectrum contains also a signal from the surrounding

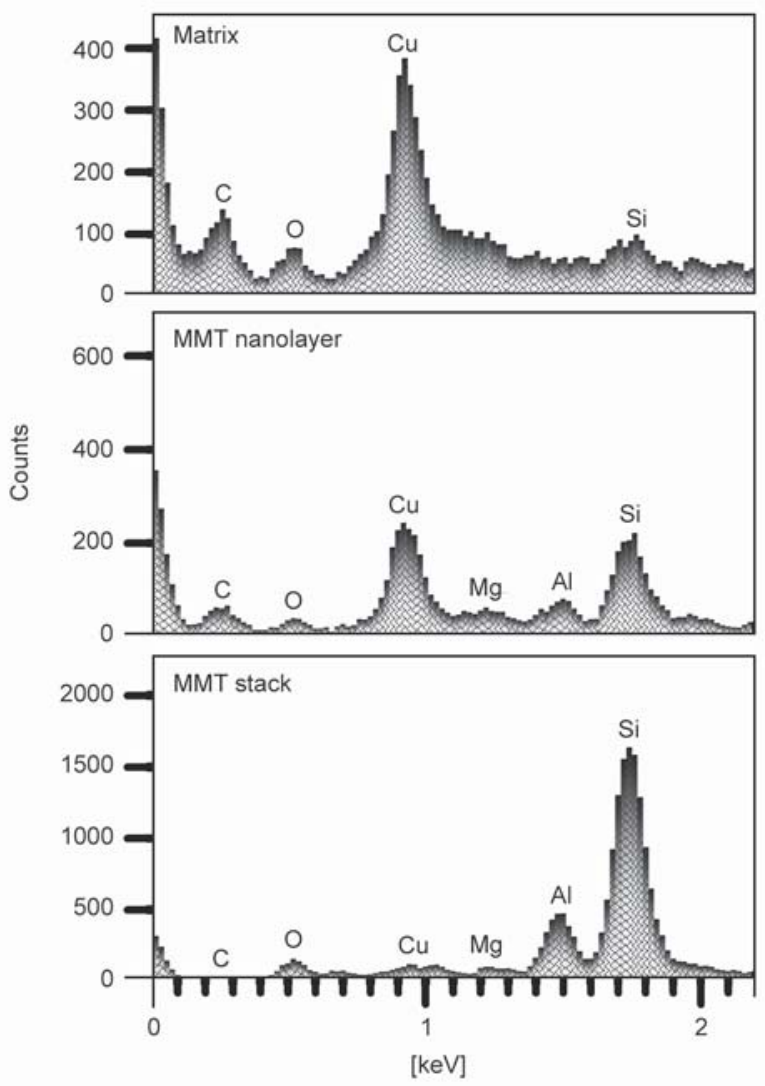

Figure 5. EDX spectra of the studied nanocomposites taken from $2 \mathrm{~nm}$ spots in diameter at focusing electron beams at polymer matrix, at single MMT nanolayer or at MMT stack. Signal from $\mathrm{Cu}$ is not related to the sample. matrix. When a nanolayer was oriented in parallel to electron beam, it provided the distinguishable contrast in a TEM image and, correspondingly, a rather strong signal of its basic elements in the EDX spectra. Naturally, the thicker MMT stacks provide the stronger signal from their elements $(\mathrm{Si}, \mathrm{Al}, \mathrm{Mg})$. Nevertheless, a signal from the polymer matrix is present in the spectra as well. On the other hand, a minor signal from MMT comprising elements is still present in the matrix spectra since the electron beam may cross some MMT layers resided at obtuse angle, which are not clearly seen in TEM image. It is noteworthy that the electron scattering results also in the signal from the supporting copper grid used for TEM specimen that explains manifesting $\mathrm{Cu}$ peaks in the EDX spectra.

\subsection{Differential scanning calorimetry}

Figure 6 shows an example of DSC curves obtained in air medium for the cured samples of the indicated nanocomposite with $0.5 \% \mathrm{MMT}$ at four successive heating cycles up to $370,400,430$ and $450^{\circ} \mathrm{C}$. One can see the natural increase in heat capacity with temperature, heat capacity steps at $T_{\mathrm{g}}$ and then exothermic effects associated with partial post-curing and the onset of the degradation processes. A gradual increasing $T_{\mathrm{g}}$ in the successive heating cycles, due to a gradual proceeding of post-curing process, can be seen. High crosslink density of matrix network resulted in strongly suppressed mobility and, therefore, very small heat capacity steps and low mechanical losses

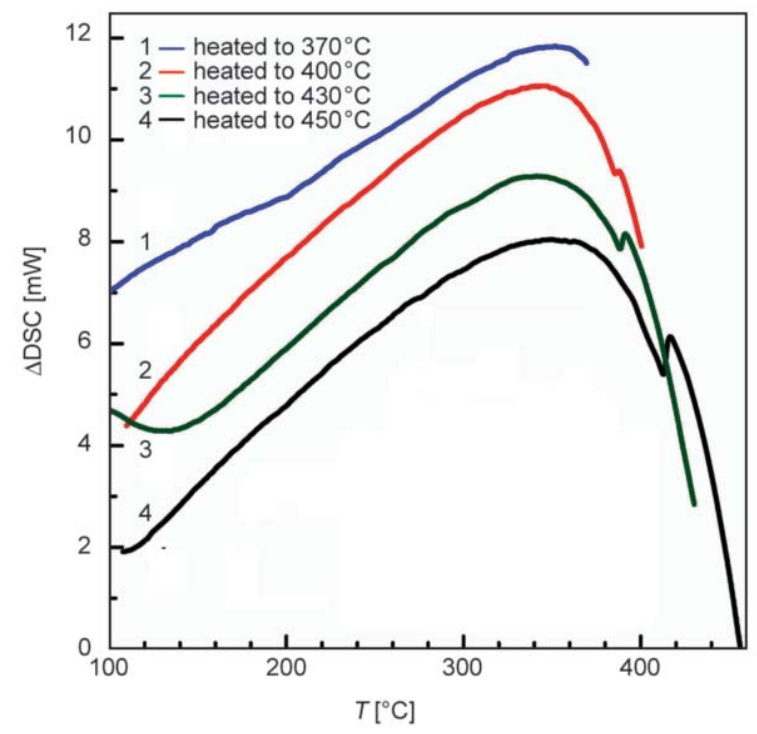

Figure 6. DSC curves obtained for the BAPhN/MMT1.30 E(0.5\%). 
level (see next section) in the glass transition. It is noteworthy that for the cured neat matrix, DSC measurements gave $T_{\mathrm{g}}=366^{\circ} \mathrm{C}$ which after heating to $430^{\circ} \mathrm{C}$ increased only to $373^{\circ} \mathrm{C}$, i.e., effect of post-curing is small. However, for the indicated nanocomposite with $0.5 \%$ MMT $T_{\mathrm{g}}=386^{\circ} \mathrm{C}$ for cured samples and may increase up to $416^{\circ} \mathrm{C}$ after post-curing (Figure 6). Nevertheless, information obtained from DSC is rather limited for these polymer systems, and dynamic mechanical analysis turned out to be much more informative for their characterization.

\subsection{Dynamic mechanical behavior: relaxation and elastic properties}

At the first scanning up to $430^{\circ} \mathrm{C}$, DMA results for initial, cured neat $\mathrm{BAPhN}$ matrix and studied nanocomposites were similar to the earlier results obtained for the BAPhN/MMT(0.5\%) [37]: in all cases, glass transition temperature $T_{\mathrm{g}}$, as estimated by $\tan \delta$ peak maximum temperature, was equal to about 380 $390^{\circ} \mathrm{C}$. The relaxation spectra of all cured samples are not presented here; one example only is shown in Figure 8a. The influence of nanoparticles on matrix dynamics was negligibly small in this case of these partially cross-linked networks.

The relaxation spectra changed cardinally at the second scanning, i.e., after post-curing procedure by heating up to $430^{\circ} \mathrm{C}$. DMA results, tan $\delta$, loss modulus $E^{\prime \prime}$ and dynamic modulus $E^{\prime}$ versus temperature dependencies, obtained for post-cured neat matrix and nanocomposites under study, are represented in Figure 7 and Table 1.

First of all, these nanocomposites with the most densely cross-linked heterocyclic matrix (PD after post-cuing increased from 89 to ca. 95\% [37]) are characterized with strongly enhanced thermal characteristics and sharply decreased mechanical losses level over the temperature range under study. Thus, maximal $\tan \delta$ in the glass transition was equal to ca. 0.2 for the cured samples (Figure 8a) which decreased down to about (0.04-0.05) for the post-cured ones (Figure 7). Despite a very low level of mechanical losses, the mechanical spectra demonstrate distinctly three broad overlapping relaxation regions, sub- $T_{\mathrm{g}}$ relaxations at $\sim 50-100$ and $200-250^{\circ} \mathrm{C}$, and broad glass transition range extending, on the whole, from about 400 to $570{ }^{\circ} \mathrm{C}$.

Figure 7 shows a single, broad glass transition peak with $T_{\mathrm{g}}=446^{\circ} \mathrm{C}$ for the post-cured neat BAPhN matrix. Another relaxation spectra are observed for the nanocomposites with different MMT contents. The common feature of these changes is not only increasing $T_{\mathrm{g}}$, up to $455-570^{\circ} \mathrm{C}$ ("constrained dynamics effects", see, e.g. the reviews $[51,52]$ describing this phenomenon) but also the complicated, dissimilar spectral contours. They show arising of the pronounced and different dynamic heterogeneity in the composites' glass transitions, e.g. manifesting their doublet structure or three-four overlapping peaks. The presence of matrix nanovolumes with relatively slight influence of MMT on the matrix $\left(T_{\mathrm{g}} \approx 460^{\circ} \mathrm{C}\right)$ and with different 'constrained dynamics' effects ( $T_{\mathrm{g}} \approx 470,500,520,530-540$ or $570{ }^{\circ} \mathrm{C}$, see Table 1$)$ are observed. It should be mentioned that the highest-temperature relaxation peak may be discerned sometimes only in the $E^{\prime \prime}(T)$ plot because of limiting the experimental DMA possibilities up to $570{ }^{\circ} \mathrm{C}$ and possible manifesting this relaxation in the $\tan \delta(T)$ plot outside this temperature range.

Analysis of the relaxation spectra of nanocomposites indicates their following peculiarities (Figure 7, Table 1). Embedding $0.03 \%$ MMT only into the matrix results in small suppressive impact of individual MMT nanolayers (see above) on dynamics of the main part of matrix: $\tan \delta$ maximum for one glass transition peak shifts from 446 to $460^{\circ} \mathrm{C}$. However, the second, very narrow peak at $529^{\circ} \mathrm{C}$ arises, which characterizes, probably, $T_{\mathrm{g}}$ in interfacial nanovolumes localized directly near single MMT nanolayers. After introducing $0.1 \% \mathrm{MMT}$, the relaxation picture became more complicated, and the spectra include strongly overlapping peaks at ca. 460, 500 and $540^{\circ} \mathrm{C}$ within the glass transition range. It means that the satisfactory exfoliation of the modified MMT nanolayers in the BAPhN matrix (see Figure 4) provides the large interfacial area and their rather large influence on matrix dynamics even at ultralow nanofiller contents. It is noteworthy that we have revealed earlier the largest influence of ultralow contents of nanofillers (POSS, MMT, embedded $\mathrm{SiO}_{2}$ units) on dynamics of Cyanate Ester Resin-based matrix [24, 48-50].

Double structure of the glass transition peak with the maxima at 455 and $540{ }^{\circ} \mathrm{C}$ is observed for the nanocomposite with $0.5 \%$ MMT. The most interesting glass transition manifestation was registered for the nanocomposite with $1 \%$ MMT: it is the complicated relaxation consisting of four overlapping peaks with close intensities and the maxima at 460, 493, 520, and $570{ }^{\circ} \mathrm{C}$. Of importance, this pronounced dynamic 

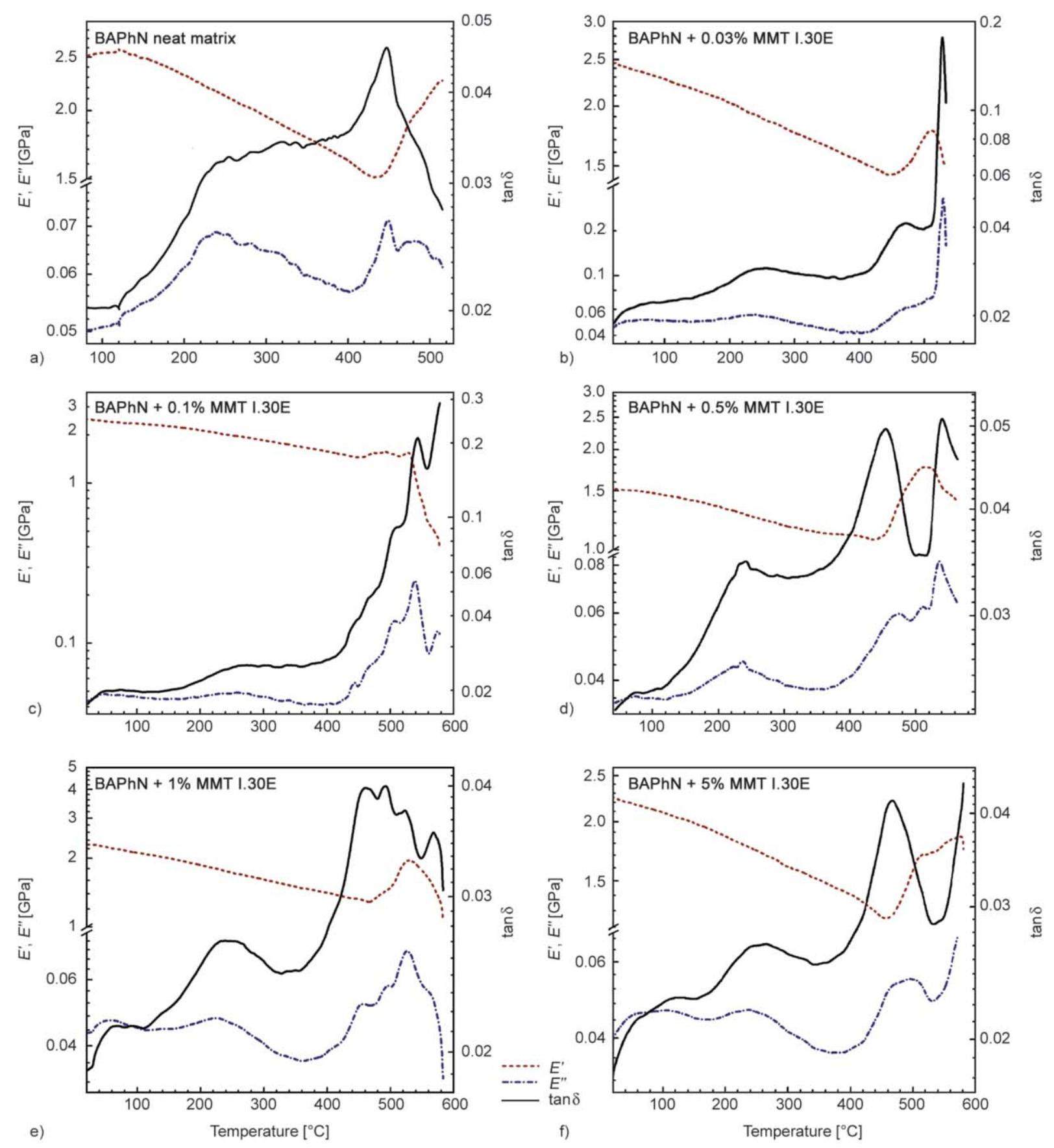

Figure 7. DMA data $(1 \mathrm{~Hz})$ obtained for post-cured samples of the neat BAPhN matrix and BAPhN-based nanocomposites with a) 0 , b) 0.03 , c) 0.1 , d) 0.5 , e) 1.0 , and f) $5.0 \mathrm{wt} \%$ MMT I.30E.

heterogeneity turned out to be in a good accordance with the nanostructural data (Figure 4). Just in this case, we observed simultaneous manifestation of different MMT exfoliation degrees including the single MMT layers, two-layers and five-ten layers MMT stacks in the matrix within $\sim 100 \mathrm{~nm}$ TEM image. At last, at 2\% and especially at 5\% MMT in the nanocomposites, the glass transition is simplified, and only doublet structure of the $T_{\mathrm{g}}$ relaxation peak is observed, at 460 and $539^{\circ} \mathrm{C}$ in the former case, and only at $470{ }^{\circ} \mathrm{C}$ at $5 \%$ MMT (Table 1 ). It may be understood, again, from the structural viewpoint. The degree of dispersion (exfoliation) of initial MMT stacks sharply decreases in these cases. Therefore, only rather thick MMT stacks-containing nanovolumes and matrix nanovolumes relatively 'free' from the nanofiller are present basically in the nanocomposites (Figure 4). It means that the interfacial area 
Table 1.The post-cured BAPhN/MMT I.30E nanocomposites (DMA, $1 \mathrm{~Hz}$ ): glass transition temperature and dynamic modulus values.

\begin{tabular}{|c|c|c|c|c|c|c|c|}
\hline \multirow{2}{*}{$\begin{array}{c}\text { MMT I.30E } \\
\text { [wt \%] }\end{array}$} & \multirow{2}{*}{$\begin{array}{c}T_{\mathrm{g}} \\
{\left[{ }^{\circ} \mathbf{C}\right]}\end{array}$} & \multicolumn{6}{|c|}{$\begin{array}{c}E^{\prime} \text { at } \\
\text { [GPa] }\end{array}$} \\
\hline & & $20^{\circ} \mathrm{C}$ & $100^{\circ} \mathrm{C}$ & $200^{\circ} \mathrm{C}$ & $300^{\circ} \mathrm{C}$ & $400^{\circ} \mathrm{C}$ & $500^{\circ} \mathrm{C}$ \\
\hline 0 & 446 & 2.5 & 2.5 & 2.3 & 1.9 & 1.6 & 2.1 \\
\hline 0.03 & 460,529 & 2.5 & 2.3 & 2.0 & 1.8 & 1.5 & 1.7 \\
\hline 0.1 & $460,500,540$ & 2.5 & 2.4 & 2.1 & 1.9 & 1.6 & 1.5 \\
\hline 0.5 & 455,540 & 2.1 & 2.0 & 1.9 & 1.6 & 1.5 & 2.2 \\
\hline 1 & $460,493,520,570$ & 2.3 & 2.1 & 1.9 & 1.6 & 1.4 & 1.5 \\
\hline 2 & 460,539 & 2.4 & 2.3 & 2.2 & 1.9 & 1.7 & 1.9 \\
\hline 5 & 470 & 2.3 & 2.1 & 1.9 & 1.6 & 1.4 & 1.8 \\
\hline
\end{tabular}

in these nanocomposites and constrained dynamics effects may be much less than those at low MMT contents.

On the whole, $T_{\mathrm{g}}$ values obtained by DMA were mainly higher than those measured by DSC. Two reasons may explain this discrepancy: (a) a difference in
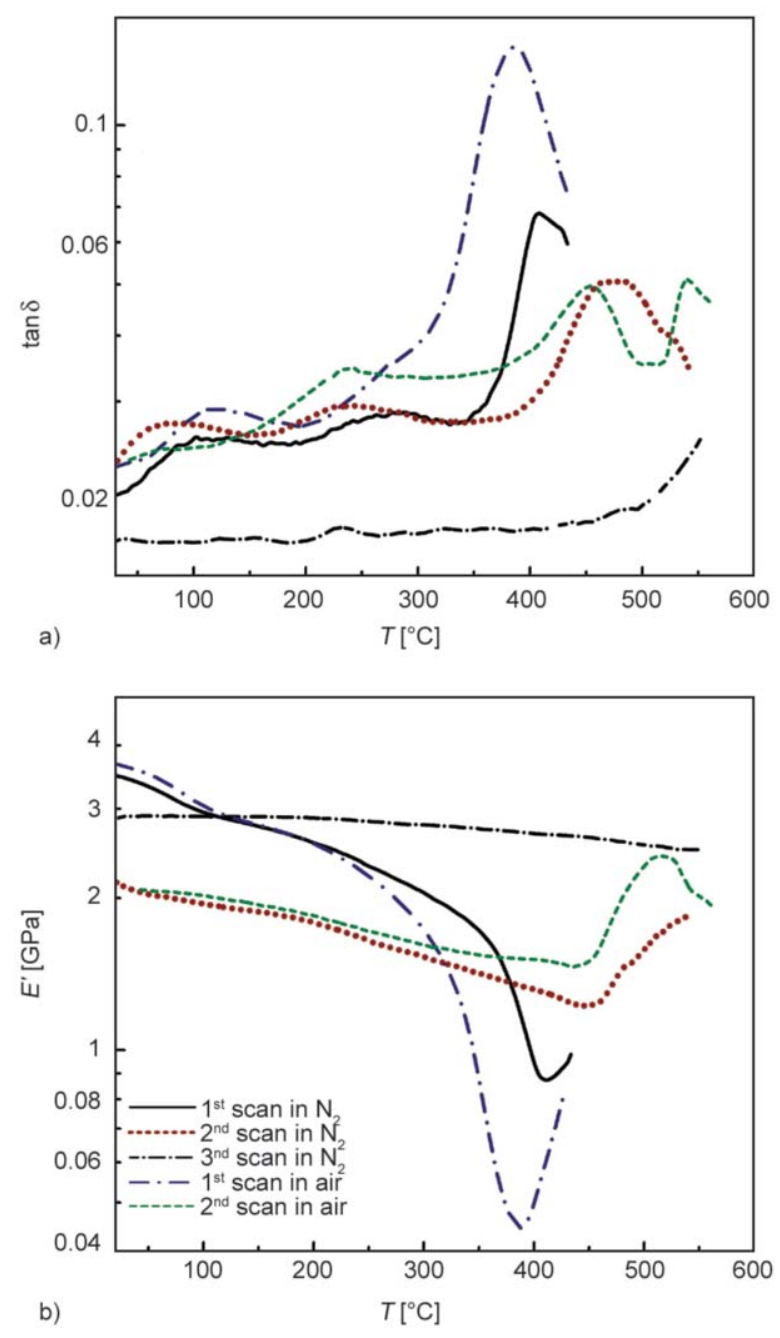

Figure 8. DMA data (1Hz): $\tan \delta(T)$ (a) and $E^{\prime}(T)$ dependencies (b) obtained under air and $\mathrm{N}_{2}$ atmosphere for the BAPhN/MMT I.30E(0.5\%) nanocomposite. time conditions of the experiments (frequency of $1 \mathrm{~Hz}$ in DMA and the equivalent frequency of $\sim 10^{-2} \mathrm{~Hz}$ in the DSC experiments), and (b) a difference in post-curing degrees attained because of scanning with the rates of 3 and $20^{\circ} \mathrm{C} / \mathrm{min}$ in the DMA and DSC experiments, respectively.

For post-cured composites, dynamic modulus $E^{\prime}(T)$ plots show (2.1-2.5) GPa values at room temperature, slow decreasing $E^{\prime}$ with temperature but its small increase at $T>430{ }^{\circ} \mathrm{C}$ (maximal temperature of the first scanning). Then, $E^{\prime}$ values decrease again at $T>500{ }^{\circ} \mathrm{C}$ due to thermo-oxidative degradation of the materials. Of interest, for the $\mathrm{BAPhN} /$ $\operatorname{MMT}(0.5 \%)$ nanocomposite $E^{\prime}$ at $500^{\circ} \mathrm{C}$ is not less than that at $20^{\circ} \mathrm{C}$ (Table 1 ).

Of special interest for some applications, e.g. in the aerospace field, is studying the comparative properties of materials at high temperatures in air and inert (oxygen-free) atmospheres. It is most important for organic materials, including polymers. In [37], we performed the comparative DMA measurements for BAPhN/POSS nanocomposite when measuring in air atmosphere and under the flowing nitrogen medium. Three heating cycles were carried out: the first cycle from 20 to $430^{\circ} \mathrm{C}$ characterized the properties of the cured composite; the second one, from 20 to $570^{\circ} \mathrm{C}$, estimated the post-cured state of this sample, and then, the third, repeat heating cycle from 20 to $570{ }^{\circ} \mathrm{C}$ was carried out in $\mathrm{N}_{2}$ medium only, in the absence of oxidizing processes. At the third scanning the total suppression of relaxation spectrum, the disappearance of glass transition, and invariable value of dynamic modulus $E^{\prime} \approx 3.2 \mathrm{GPa}$ over the temperature range from 20 to $\sim 600^{\circ} \mathrm{C}$ were observed [37]. The latter fact was registered, probably, for polymers for the first time.

Herein, the similar experiments were performed for the BAPhN/MMT(0.5\%) nanocomposite. Figure 8 
shows that at the first scanning the influence of medium on relaxation and elastic properties of this nanocomposite is negligibly small only up to $200-250^{\circ} \mathrm{C}$, however, then the $\tan \delta(T)$ and $E^{\prime}(T)$ curves diverge: the steep rise of relaxation starts from $\sim 300^{\circ} \mathrm{C}$ in air medium but from $\sim 350^{\circ} \mathrm{C}$ in nitrogen atmosphere. $T_{\mathrm{g}}$ values at the maxima of glass transition peaks are registered for this cured sample at $\sim 380$ and $400{ }^{\circ} \mathrm{C}$, respectively (Figure $8 \mathrm{a}$ ). Simultaneously, modulus value decreases at $T_{\mathrm{g}}$ down to 0.45 and $0.9 \mathrm{GPa}$ in air and nitrogen mediums, increasing, however, to some extent at heating to $430^{\circ} \mathrm{C}$ due to post-curing proceeding (Figure $8 \mathrm{~b}$ ). The second scanning characterizes already the post-cured state of this nanocomposite. Two $T_{\mathrm{g}} \mathrm{s}$, at 455 and $540^{\circ} \mathrm{C}$, are registered in air medium but only one $T_{\mathrm{g}} \approx 470^{\circ} \mathrm{C}$ in the inert atmosphere. There is no large dropping of modulus with temperature in both mediums; it retains at the level of $\sim 2 \mathrm{GPa}$, and slightly increases in the region of $430-570^{\circ} \mathrm{C}$, although at $T>500^{\circ} \mathrm{C}$ some decreasing $E^{\prime}$ starts in air medium because of the onset of thermo-oxidative processes (see next section). At last, similar to the result obtained for POSS-containing nanocomposite [37], at the third scanning in $\mathrm{N}_{2}$ atmosphere we observe the total suppression of relaxation spectrum, the disappearance of glass transition, and invariable value of dynamic modulus $E^{\prime} \approx 3 \mathrm{GPa}$ over the temperature range from 20 to $\sim 600^{\circ} \mathrm{C}$ (Figure 8). To our knowledge, the latter fact was registered in [37] and herein for polymers for the first time.

In conclusion of this section, two comments may be done. First, at second scanning in $\mathrm{N}_{2}$ medium we, as earlier [37], do not reveal the dynamic heterogeneity within the glass transition: only the shift of relaxation peak from $\sim 400$ to $\sim 470^{\circ} \mathrm{C}$ is observed. This result suggests that the relaxations at temperatures $T>$ $50{ }^{\circ} \mathrm{C}$ within glass transition of the studied phthalonitrile nanocomposites may be associated in the experiments in air medium, to some extent, with the onset of thermal-oxidative degradation of matrix network. Secondly, the measurements have been performed herein with the $0.5 \mathrm{~mm}$-thickness films. It may be suggested that in case of thick nanocomposite construction, underwent to short-term high-temperature heating, the influence of oxidizing (air) medium may be less pronounced than in our experiments.

\subsection{Thermal stability and thermo-oxidative degradation}

Thermal stability and thermo-oxidative degradation were studied using TGA and DTG analyses under air atmosphere and inert $\left(\mathrm{N}_{2}\right)$ medium, respectively, at temperatures between 20 and $700-900^{\circ} \mathrm{C}$ for the neat matrix and three nanocomposites, with $0.5,2$ and $5 \%$ $\mathrm{BAPhN} / \mathrm{MMT}$. The results obtained are represented in Figures 9 and 10 and in Table 2. Very high thermal stability of these materials is obvious.

As shown in Figure 9a, TGA curves obtained for the neat matrix are characterized with the total stability
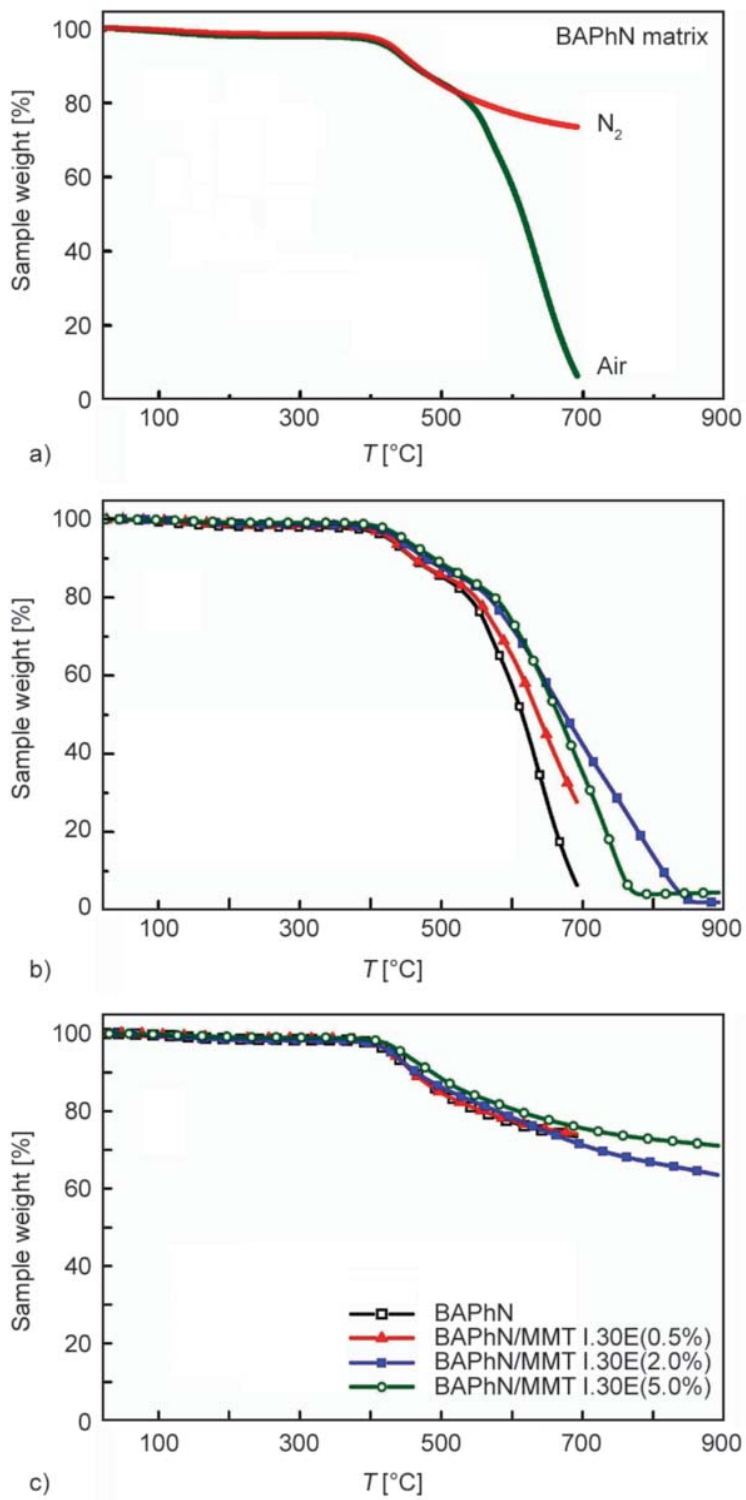

Figure 9. TGA data obtained for the neat $\mathrm{BAPhN}$ matrix in air and $\mathrm{N}_{2}$ mediums (a), and for the neat BAPhN matrix and three indicated nanocomposites in air (b) and $\mathrm{N}_{2}$ medium (c). 
up to $300-350^{\circ} \mathrm{C}$ where mass loss of $1.5-2 \mathrm{wt} \%$ is absorbed water removal. Slight thermal degradation starts from $\sim 430^{\circ} \mathrm{C}$ when mass loss of $\sim 5 \%$ is registered. The process of thermal degradation at this early stage does not depend practically on the medium up to $\sim 550^{\circ} \mathrm{C}$. About $20 \%$ mass is lost at the latter temperature at heating both in air and nitrogen mediums but the films retain the satisfactory integrity at this temperature. Meanwhile, from the temperature $T \approx 550^{\circ} \mathrm{C}$, as the 'temperature bifurcation point', TGA plots, obtained in air and nitrogen mediums sharply diverge due to 'switching on' of intense thermo-oxidative degradation processes in air medium. As a result, air curve in Figure 9 goes abruptly

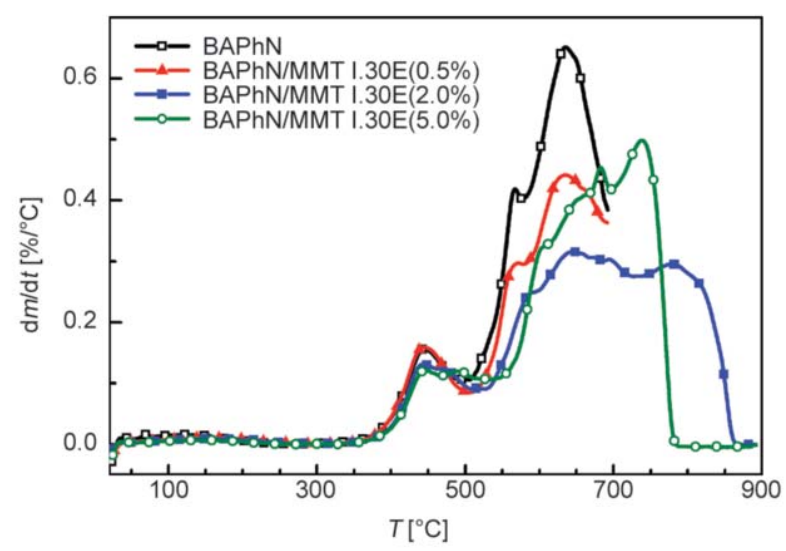

Figure 10. DTG data obtained for the neat BAPhN matrix and three indicated nanocomposites in air medium. down, whereas the additional destructive processes are insignificant in inert atmosphere up to $700^{\circ} \mathrm{C}$. Char residues at $\sim 700^{\circ} \mathrm{C}$ are equal to 7 and $73 \mathrm{wt} \%$ in air and $\mathrm{N}_{2}$, respectively. DTG plots show for the neat matrix one slight and two intense overlapping peaks with the temperatures of maximal degradation rate, $T_{\mathrm{d} \text { max }}$, at $446^{\circ} \mathrm{C}$, and 567 and $636^{\circ} \mathrm{C}$ in air atmosphere but only one peak of slight degradation with the $T_{\mathrm{d} \text { max }}=445^{\circ} \mathrm{C}$ in $\mathrm{N}_{2}$ medium (Figure 10, Table 2).

For the nanocomposites with $0.5,2$ and 5\% MMT, Figures 9 and 10 and Table 2 give the following results (for the latter two composites, the measurements were performed up to ca. $900^{\circ} \mathrm{C}$ ). In the measurements in $\mathrm{N}_{2}$ medium, no substantial differences in thermal stability of the neat matrix and the nanocomposites were revealed, although the maximal char residues at 750 and ca. $900{ }^{\circ} \mathrm{C}, 74$ and $71 \%$, were registered for the composite with 5\% MMT. Moreover, these composite films retained the satisfactory integrity under these conditions. Only small changes in thermal behavior arose as well after adding MMT. Meantime, incorporating MMT nanolayers resulted in increasing thermo-oxidative stability in air medium: in particular, char residue at $\sim 700^{\circ} \mathrm{C}$ increased from $7 \%$ for the neat matrix up to $45 \%$ for the composite with $2 \%$ MMT; the DTG plot for the latter shifted substantially to higher temperatures (Table 2, Figures 9 and 10).

Table 2. Thermal stability and thermal-oxidative degradation (TGA/DTG results).

\begin{tabular}{|c|c|c|c|c|c|c|c|c|c|c|}
\hline \multicolumn{11}{|c|}{ Air atmosphere } \\
\hline \multirow[t]{2}{*}{ Sample } & \multirow[t]{2}{*}{$\begin{array}{c}T_{\mathrm{d}}(\mathbf{5 \%}) \\
{\left[{ }^{\circ} \mathrm{C}\right]}\end{array}$} & \multirow[t]{2}{*}{$\begin{array}{c}T_{\mathrm{d}}(10 \%) \\
{\left[{ }^{\circ} \mathrm{C}\right]}\end{array}$} & \multirow[t]{2}{*}{$\begin{array}{c}T_{\mathrm{d}}(\mathbf{2 0} \%) \\
{\left[{ }^{\circ} \mathrm{C}\right]}\end{array}$} & \multicolumn{4}{|c|}{$\begin{array}{l}\text { Temperature of maximal degradation } \\
\text { rate, } T_{\mathrm{d} \text { max }} \text {, at the stage } \\
{\left[{ }^{\circ} \mathrm{C}\right]}\end{array}$} & \multicolumn{3}{|c|}{$\begin{array}{c}\text { Char residue at } T: \\
{[w \%]}\end{array}$} \\
\hline & & & & I & II & III & IV & $690^{\circ} \mathrm{C}$ & $750^{\circ} \mathrm{C}$ & $890^{\circ} \mathrm{C}$ \\
\hline $\mathrm{BAPhN}$ & 426 & 460 & 539 & 446 & 567 & 636 & - & 7 & - & - \\
\hline BAPhN/MMT I.30E (0.5\%) & 429 & 462 & 549 & 445 & 569 & 636 & - & 28 & - & - \\
\hline BAPhN/MMT I.30E (2.0\%) & 437 & 479 & 566 & 444 & 589 & 645 & 780 & 45 & 28 & $\sim 3$ \\
\hline BAPhN/MMT I.30E (5.0\%) & 446 & 489 & 576 & 446 & 603 & 683 & 738 & 39 & 12 & $\sim 5$ \\
\hline \multicolumn{11}{|c|}{ Nitrogen atmosphere } \\
\hline \multirow[t]{2}{*}{ Sample } & \multirow[t]{2}{*}{$\begin{array}{c}T_{\mathrm{d}}(5 \%) \\
{\left[{ }^{\circ} \mathrm{C}\right]}\end{array}$} & \multirow[t]{2}{*}{$\begin{array}{c}T_{\mathrm{d}}(10 \%) \\
{\left[{ }^{\circ} \mathrm{C}\right]}\end{array}$} & \multirow[t]{2}{*}{$\begin{array}{c}T_{\mathrm{d}}(20 \%) \\
{\left[{ }^{\circ} \mathrm{C}\right]}\end{array}$} & \multicolumn{4}{|c|}{$\begin{array}{c}\text { Temperature of maximal } \\
\text { degradation rate, } T_{\mathrm{d} \text { max }} \text {, at the stage } \\
{\left[{ }^{\circ} \mathrm{C}\right]}\end{array}$} & \multicolumn{3}{|c|}{$\begin{array}{c}\text { Char residue at } T: \\
{[\mathrm{wt} \%]}\end{array}$} \\
\hline & & & & I & & & III & $690^{\circ} \mathrm{C}$ & $750^{\circ} \mathrm{C}$ & $890^{\circ} \mathrm{C}$ \\
\hline BAPhN & 432 & 463 & 557 & 445 & & & - & 73 & - & - \\
\hline BAPhN/MMT I.30E $(0.5 \%)$ & 431 & 461 & 559 & 448 & & & - & 74 & - & - \\
\hline BAPhN/MMT I.30E (2.0\%) & 430 & 463 & 577 & 444 & & & - & 72 & 69 & 64 \\
\hline BAPhN/MMT I.30E (5.0\%) & 445 & 487 & 608 & 449 & & & 613 & 76 & 74 & 71 \\
\hline
\end{tabular}

Note. The $T_{\mathrm{d}}$ and $T_{\mathrm{d} \text { max }}$ are the temperatures corresponding to the indicated mass losses $(5,10$, or $20 \%$ ) and the maxima of DTG peaks, respectively. 
Enhancing thermal stability in MMT-containing nanocomposites has been observed before in numerous investigations (see, e.g. review article [53]). The origin of this effect is, obviously, rather complicated: it may be connected, presumably, with changes in degradation kinetics due to the barrier effect, suppressed dynamics in the matrix, restricting of oxygen diffusion, etc. [53].

Previously, TGA investigation of non-isothermal decomposition of Cyanate Ester Resin (CER) matrix and CER/amino-MMT nanocomposites (containing only triazine and benzene rings) in $\mathrm{N}_{2}$ medium showed that the degradation occurred most intensely at $430-500^{\circ} \mathrm{C}$ : almost half of mass was lost [24]. Korshak et al. [54] showed that this degradation process corresponded to breakage of bonds between the phenyl and triazine rings in CER followed by intensive decomposition of cyanurate skeleton. We suggest, therefore, that such ultrahigh stability in nitrogen atmosphere is associated with leading role of extremely thermostable phthalocyanine macrocycles as the main constituent of $\mathrm{BAPhN}$ network.

\section{Conclusions}

Synthesis and research of structure, dynamics and properties were performed for a series of thermostable phthalonitrile nanocomposites containing different contents $(0.03-5 \mathrm{wt} \%)$ of organically-modified montmorillonite nanoparticles. Bisphenol A phthalonitrile $(\mathrm{BAPhN})$ and amino-modified montmorillonite (MMT I.30E) were used for the synthesis. The samples of hybrid nanocomposites cured at $260-300^{\circ} \mathrm{C}$ with the polymerization degrees of 83 $89 \%$, and the nanocomposites post-cured by heating up to $430^{\circ} \mathrm{C}$, with the maximal polymerization degree of ca. $95 \%$, were studied.

New data regarding the complicated molecular structure of BAPhN matrix and the supposition about the leading contribution of phthalocyanine macrocycles to the matrix molecular structure were obtained by using far- and mid-IR spectroscopy. Depending on the MMT I.30E content, different exfoliation degrees of its stacks, down to single nanolayers, were shown by TEM and validated by EDX spectroscopy. The 'constrained dynamics' effects, caused by embedding reactive nanoparticles, were registered by DMA and in the far-IR spectra. The nanostructure-relaxation properties relationships could be traced. DMA demonstrates the pronounced dynamic heterogeneity in the glass transition of nanocomposites. On the whole, $T_{\mathrm{g}}$ (DMA) values varied from $\sim 460$ to $570^{\circ} \mathrm{C}$, and maximal dynamic modulus $E^{\prime}=2.5 \mathrm{GPa}$ at room temperature was registered for the nanocomposites post-cured in air atmosphere. The comparative DMA and TGA measurements were performed in air and $\mathrm{N}_{2}$ mediums over the temperature ranges from 20 to $\sim 600$ and $900{ }^{\circ} \mathrm{C}$, respectively. It was shown that the most substantial influence of air medium, due to the onset of thermo-oxidative degradation, started from $\sim 500{ }^{\circ} \mathrm{C}$. This process is inhibited partially by adding of $2 \%$ MMT I.30E : at $690{ }^{\circ} \mathrm{C}$ char residue increases from 7 to $45 \%$. A relatively satisfactory thermal stability in inert $\left(\mathrm{N}_{2}\right)$ medium was observed up to ca. $900{ }^{\circ} \mathrm{C}$ : for the nanocomposite with 5\% MMT I.30E char residue achieved $71 \%$, and the composite films retained satisfactory integrity. Total suppression of glass transition and invariability of modulus value $\left(E^{\prime} \approx 3 \mathrm{GPa}\right)$ over the temperature range from 20 to $600{ }^{\circ} \mathrm{C}$ were observed for the BAPhN/MMT $1.30 \mathrm{E} \quad(0.5 \%)$ nanocomposite after its high temperature treatment in oxygen-free atmosphere. For this nanocomposite, the equality of modulus $E^{\prime}$ values at 30 and $500^{\circ} \mathrm{C}$ was registered also under air conditions. Due to their ultrahigh thermal stability and other properties, the nanocomposites developed may be considered as promising candidate materials for the application under the extreme conditions in aerospace, submarine, microelectronic industries, and in 'hot zones' of different constructions.

\section{Acknowledgements}

TEM and EDXS characterizations were performed at Ioffe Institute using equipment of the Federal Joint Research Center 'Material science and characterization in advanced technology' supported by the Ministry of Education and -Science of the Russian Federation (id RFMEFI62117X0018).

\section{References}

[1] Kim H., Abdala A. A., Macosko C. W.: Graphene/polymer nanocomposites. Macromolecules, 43, 6515-6530 (2010). https://doi.org/10.1021/ma100572e

[2] Karger-Kocsis J., Mahmood H., Pegoretti A.: Recent advances in fiber/matrix interphase engineering for polymer composites. Progress in Materials Science, 73, 1-43 (2015).

https://doi.org/10.1016/j.pmatsci.2015.02.003 
[3] Akpan E. I., Shen X., Wetzel B., Friedrich K.: Design and synthesis of polymer nanocomposites. in 'Polymer composites with functionalized nanoparticles' (eds.: Pielichowski K., Majka T.) Elsevier, Amsterdam 47-83 (2019).

https://doi.org/10.1016/b978-0-12-814064-2.00002-0

[4] Jlassi K., Chehimi M. M., Thomas S.: Clay-polymer nanocomposites. Elsevier, Amsterdam (2017).

[5] Mittal V.: Polymer layered silicate nanocomposites: A review. Materials, 2, 992-1057 (2009).

https://doi.org/10.3390/ma2030992

[6] Nair C. P. R., Mathew D., Ninan K. N.: Cyanate ester resins, recent developments. Advances in Polymer Science, 155, 1-99 (2001).

https://doi.org/10.1007/3-540-44473-4_1

[7] Gilman J. W., Harris R., Hunter D.: Cyanate ester clay nanocomposites: Synthesis and flammability studies. in ' $44^{\text {th }}$ International SAMPE Symposium/Exihibition, Long Beach, USA’ 1408-1423 (1999).

[8] Gilman J. W., Harris R. H., Jackson C. L., Morgan A. B., Brassell L. D., Hunter D. L.: Phenolic cyanate ester clay nanocomposites: Effect of ammonium ion structure on flammability and nano-dispersion. in 'Polymeric Materials: Science and Engineering (PMSE). Spring Meeting, San Francisco, USA' Vol 82, 276-277 (2000).

[9] Morgan A. B., Gilman J. W.: Characterization of polymer-layered silicate (clay) nanocomposites by transmission electron microscopy and X-ray diffraction: A comparative study. Journal of Applied Polymer Science, 87, 1329-1338 (2003).

https://doi.org/10.1002/app.11884

[10] Ganguli S., Dean D., Jordan K., Price G., Vaia R.: Mechanical properties of intercalated cyanate ester-layered silicate nanocomposites. Polymer, 44, 1315-1319 (2003).

https://doi.org/10.1016/S0032-3861(02)00709-7

[11] Ganguli S., Dean D., Jordan K., Price G., Vaia R.: Chemorheology of cyanate ester-organically layered silicate nanocomposites. Polymer, 44, 6901-6911 (2003). https://doi.org/10.1016/j.polymer.2003.08.031

[12] Wooster T. J., Abrol S., MacFarlane D. R.: Cyanate ester polymerization catalysis by layered-silicates. Polymer, 45, 7845-7852 (2004).

https://doi.org/10.1016/j.polymer.2004.09.040

[13] Kim D. S., Lee K. M.: Polymerization kinetics and thermal properties of dicyanate/clay nanocomposites. Journal of Applied Polymer Science, 92, 1955-1960 (2004). https://doi.org/10.1002/app.20224

[14] Kissounko D. A., Deitzel J. M., Doherty S. P., Shah A., Gillespie J. W.: Understanding the role of clay silicate nanoparticles with organic modifiers in thermal curing of cyanate ester resin. European Polymer Journal, 44, 2807-2819 (2008).

https://doi.org/10.1016/j.eurpolymj.2008.06.034

[15] Siddiqui A. O., Sudher P., Murthy B. V. S. R.: Cure kinetics modeling of cyanate-ester resin system. Thermochimica Acta, 554, 8-14 (2013).

https://doi.org/10.1016/j.tca.2012.12.009
[16] John B., Nair C. P. R., Ninan K. N.: Effect of nanoclay on the mechanical, dynamic mechanical and thermal properties of cyanate ester syntactic foams. Materials Science and Engineering: A, 527, 5435-5443 (2010). https://doi.org/10.1016/j.msea.2010.05.016

[17] Lin Y., Song M., Stone C. A., Shaw S. J.: A comprehensive study on the curing kinetics and network formation of cyanate ester resin/clay nanocomposites. Thermochimica Acta, 552, 77-86 (2013). https://doi.org/10.1016/j.tca.2012.11.009

[18] Wooster T. J., Abrol S., MacFarlane D. R.: Rheological and mechanical properties of percolated cyanate ester nanocomposites. Polymer, 46, 8011-8017 (2005).

https://doi.org/10.1016/j.polymer.2005.06.106

[19] Meng J., Hu X., Boey F. Y. C., Li L.: Effect of layered nano-organosilicate on the gel point rheology of bismaleimide/diallylbisphenol A resin. Polymer, 46, 27662776 (2005).

https://doi.org/10.1016/j.polymer.2004.11.087

[20] Zhang J., Hu S., Zhan G., Tang X., Yu Y.: Biobased nanocomposites from clay modified blend of epoxidized soybean oil and cyanate ester resin. Progress in Organic Coatings, 76, 1683-1690 (2013). https://doi.org/10.1016/j.porgcoat.2013.07.017

[21] Kim D. S., Lee K. M.: Mechanical properties and structures of dicyanate-clay nanocomposites. Journal of Applied Polymer Science, 90, 2629-2633 (2003).

https://doi.org/10.1002/app.12893

[22] Bershtein V. A., Fainleib A. M., Pissis P., Bei I. M., Dalmas F., Egorova L. M., Gomza Yu. P., Kripotou S., Maroulas P., Yakushev P. N.: Polycyanurate-organically modified montmorillonite nanocomposites: Structure-dynamics-properties relationships. Journal of Macromolecular Science Part B: Physics, 47, 555-575 (2008). https://doi.org/10.1080/00222340801955545

[23] Anthoulis G. I., Kontou E., Fainleib A. M., Bei I., Gomza Y.: Synthesis and characterization of polycyanurate/ montmorillonite nanocomposites. Journal of Polymer Science Part B: Physics, 46, 1036-1048 (2008).

https://doi.org/10.1002/polb.21436

[24] Bershtein V. A., Fainleib A. M., Egorova L. M., Gusakova K. G., Grigoyeva O. P., Kirilenko D. A., Konnikov S. G., Ryzhov V. A., Yakushev P. N., Lavrenyuk N.: The impact of ultra-low amounts of amino-modified MMT on dynamics and properties of densely cross-linked cyanate ester resins. Nanoscale Research Letters, 10, 165/1-165/15 (2015). https://doi.org/10.1186/s11671-015-0868-5

[25] McConnell V. P.: Resins for the hot zone, Part II: BMIs, CEs, benzoxazines and phthalonitriles. High-Performance Composites, 17, 43-49 (2009).

[26] Derradji M., Wang J., Liu W. B.: Phthalonitrile resins and composites. Properties and applications. Elsevier, New York (2018).

[27] Keller T. M.: Phthalonitrile resin from diphthalonitrile monomer and amine. U.S. Patent 4,408,035, USA (1983). 
[28] Keller T. M., Price T.: Amine-cured bisphenol-linked phthalonitrile resins. Journal of Macromolecular Science Part A: Chemistry, 18, 931-937 (1982). https://doi.org/10.1080/00222338208077208

[29] Keller T. M.: Phthalonitrile-based high temperature resin. Journal of Polymer Science Part A Polymer Chemistry, 26, 3199-3212 (1988).

https://doi.org/10.1002/pola.1988.080261207

[30] Lei Y., Hu G-H., Zhao R., Guo H., Zhao X., Liu X.: Preparation process and properties of exfoliated graphite nanoplatelets filled bisphthalonitrile nanocomposites. Journal of Physics and Chemistry of Solids, 73, 13351341 (2012).

https://doi.org/10.1016/j.jpcs.2012.07.003

[31] Lei Y., Zhao R., Yang X., Liu X.: Mechanical and thermal properties of graphite nanoplatelets reinforced polyarylene ether nitriles/bisphthalonitrile IPN system. Journal of Applied Polymer Science, 5, 3595-3600 (2013).

https://doi.org/10.1002/app.37754

[32] Derradji M., Ramdani N., Zhang T., Wang J., Lin Z-W., Yang M., Xu X-D., Liu W-B.: High thermal and thermomechanical properties obtained by reinforcing a bisphenol-A based phthalonitrile resin with silicon nitride nanoparticles. Materials Letters, 149, 81-84 (2015). https://doi.org/10.1016/j.matlet.2015.02.122

[33] Derradji M., Wang J., Liu W-B.: High performance ceramic-based phthalonitrile micro and nanocomposites. Materials Letters, 182, 380-385 (2016).

https://doi.org/10.1016/j.matlet.2016.06.110

[34] Derradji M., Ramdani N., Gong L-D., Wang J., Xu X-D., Lin Z-W., Henniche A., Liu W. B.: Mechanical, thermal, and UV-shielding behavior of silane surface modified $\mathrm{ZnO}$-reinforced phthalonitrile nanocomposites. Polymers for Advanced Technologies, 27, 882-888 (2016).

https://doi.org/10.1002/pat.3744

[35] Derradji M., Ramdani N., Zhang T., Wang J., Gong L-D., Xu X-D., Lin Z-W., Henniche A., Rahoma K. S., Liu W-B: Effect of silane surface modified titania nanoparticles on the thermal, mechanical, and corrosion protective properties of a bisphenol-A based phthalonitrile resin. Progress in Organic Coatings, 90, 34-43 (2016). https://doi.org/10.1016/j.porgcoat.2015.09.021

[36] Li X., Yu B., Zhang D., Lei J., Nan Z.: Cure behavior and thermomechanical properties of phthalonitrilepolyhedral oligomeric silsesquioxane copolymers. Polymers, 9, 334/1-334/13 (2017).

https://doi.org/10.3390/polym9080334

[37] Bershtein V. A., Fainleib A. M., Yakushev P. N., Kirilenko D. A., Gusakova K. G., Markina D. I., Melnychuk O. G., Ryzhov V. A.: High temperature phthalonitrile nanocomposites with silicon based nanoparticles of different nature and surface modification: Structure, dynamics, properties. Polymer, 165, 39-54 (2019). https://doi.org/10.1016/j.polymer.2019.01.020
[38] Kirilenko D. A., Dideykin A. T., Aleksenskiy A. E., Sitnikova A. A., Konnikov S. G., Vul' A. Y.: One-step synthesis of a suspended ultrathin graphene oxide film: Application in transmission electron microscopy. Micron, 68, 23-26 (2015).

https://doi.org/10.1016/j.micron.2014.08.006

[39] Sheng H., Peng X., Guo H., Yu X., Naito K., Qu X., Zhang Q.: Synthesis of high performance bisphthalonitrile resins cured with self-catalyzed 4-aminophenoxy phthalonitrile. Thermochimica Acta, 577, 17-24 (2014). https://doi.org/10.1016/j.tca.2013.12.010

[40] Kumar D., Razdan U., Gupta A. D.: Heat-resistant polymers from melt-processable bisimido-bisphthalonitriles. Journal of Polymer Science Part A: Polymer Chemistry, 31, 797-804 (1993). https://doi.org/10.1002/pola.1993.080310326

[41] Zou Y., Yang J., Zhan Y., Yang X., Zhong J., Zhao R., Liu X.: Effect of curing behaviors on the properties of poly(arylene ether nitrile) end-capped with phthalonitrile. Journal of Applied Polymer Science, 125, 38293835 (2012). https://doi.org/10.1002/app.36691

[42] Wu Z., Han J., Li N., Weng Z., Wang J., Jian X.: Improving the curing process and thermal stability of phthalonitrile resin via novel mixed curing agents. Polymer International, 66, 876-881 (2017).

https://doi.org/10.1002/pi.5328

[43] Ji S., Yuan P., Hu J., Sun R., Zeng K., Yang G.: A novel curing agent for phthalonitrile monomers: Curing behaviors and properties of the polymer network. Polymer, 84, 365-370 (2016).

https://doi.org/10.1016/j.polymer.2016.01.006

[44] Sheng H., Peng X., Guo H., Yu X., Tang C., Qu X., Zhang Q.: Synthesis and thermal properties of a novel high temperature alkyl-center-trisphenolic-based phthalonitrile polymer. Materials Chemistry and Physics, 142, 740-747 (2013). https://doi.org/10.1016/j.matchemphys.2013.08.033

[45] Laskosky M., Neal A., Keller T. M., Dominguez D., Klug C. A., Saab A. P.: Improved synthesis of oligomeric phthalonitriles and studies designed for low temperature cure. Journal of Polymer Science Part A: Polymer Chemistry, 52, 1662-1668 (2014).

https://doi.org/10.1002/pola.27161

[46] Kobayashi T.: The far infrared spectra of phthalocyanine and its metal derivatives. Spectrochimica Acta Part A: Molecular Spectroscopy, 26, 1313-1322 (1970). https://doi.org/10.1016/0584-8539(70)80037-X

[47] Bershtein V. A., Ryzhov V. A.: Far infrared spectroscopy of polymers. Advances in Polymer Science, 114, 43-121 (1994). https://doi.org/10.1007/BFb0008694 
[48] Bershtein V., Fainleib A., Egorova L., Grgoryeva O., Kirilenko D., Konnikov S., Ryzhov V., Starostenko O., Yakushev P., Yagovkina M., Saiter J-M.: The impact of ultra-low amounts of introduced reactive POSS nanoparticles on structure, dynamics and properties of densely cross-linked cyanate ester resins. European Polymer Journal, 67, 128-142 (2015).

https://doi.org/10.1016/j.eurpolymj.2015.03.022

[49] Bershtein V. A., Fainleib A. M., Kirilenko D. A., Yakushev P. N., Gusakova K. G., Lavrenyuk N., Ryzhov V. A.: Dynamics and properties of high performance amorphous cyanate ester-based subnanocomposites with ultralow silica content and quasi-regular structure. Polymer, 103, 36-40 (2016).

https://doi.org/10.1016/j.polymer.2016.09.020

[50] Bershtein V., Fainleib A., Gusakova K., Kirilenko D., Yakushev P., Egorova L., Lavrenyuk N., Ryzhov V.: Silica subnanometer-sized nodes, nanoclusters and aggregates in cyanate ester resin-based networks: Structure and properties of hybrid subnano- and nanocomposites. European Polymer Journal, 85, 375-389 (2016). https://doi.org/10.1016/j.eurpolymj.2016.10.047
[51] Granick S. (ed.): Polymers in confined environments. Advances in Polymer Science, Springer, Berlin (1999).

[52] Bershtein V. A., Yakushev P. N.: Laser-interferometric creep rate spectroscopy of polymers. in 'Polymer Characterization. Advances in Polymer Science' (eds.: Dusek K., Joanny J. F.) Springer, Berlin, Vol 230, 73-219 (2010).

https://doi.org/10.1007/12 200936

[53] Leszczyńska A., Njuguna J., Pielichowski K., Banerjee J. R.: Polymer/montmorillonite nanocomposites with improved thermal properties: Part II. Thermal stability of montmorillonite nanocomposites based on different polymeric matrixes. Thermochimica Acta, 454, 1-22 (2007).

https://doi.org/10.1016/j.tca.2006.11.003

[54] Korshak V. V., Gribkova P. N., Dmitrenko A. V., Puchin A. G., Pankratov V. A., Vinogradova S. V.: Thermal and thermal-oxidative degradation of polycyanates. Polymer Science U.S.S.R., 16, 15-23 (1974).

https://doi.org/10.1016/0032-3950(74)90111-7 